\title{
Monte Carlo Renormalization of the 3D Ising Model: Analyticity and Convergence
}

\author{
H. W. J. Blöte, J. R. Heringa, A. Hoogland, E. W. Meyer, and T. S. Smit \\ Department of Applied Physics, Delft University of Technology, Lorentzweg 1, 2628 CJ Delft, The Netherlands
}

(Received 27 November 1995)

\begin{abstract}
We review the assumptions on which the Monte Carlo renormalization technique is based, in particular, the analyticity of the block-spin transformations. On this basis, we select an optimized Kadanoff blocking rule in combination with the simulation of a $d=3$ Ising model with reduced corrections to scaling. This is achieved by including interactions with second and third neighbors. As a consequence of the improved transformation, this Monte Carlo renormalization method yields a fast convergence and a high accuracy. The results for the critical exponents are $y_{H}=2.481(1)$ and $y_{T}=1.585(3)$.

PACS numbers: 05.50.+q, 02.70.Lq, 75.10.Hk, 75.40.Mg
\end{abstract}

Applications of the Monte Carlo renormalization group (MCRG) to the three-dimensional Ising model [1-4] have become increasingly elaborate and complicated, and tend to require considerable computer resources. Nevertheless, there are still uncertainties due to the basic assumptions underlying the renormalization transformations used. In particular, we will focus on the question concerning the analyticity of the transformation, which may be related to the question whether the corrections to scaling vanish at the fixed point of the transformation [5]. In this Letter we first calculate the analytic part of a divergent observable; this demonstrates that gross nonanalyticities are normally absent. However, in general we may expect weak nonanalyticities due to corrections to scaling. Thus, second, we minimize their effects by adjusting the transformation as well as the Hamiltonian that is simulated.

The MCRG method has amply been reviewed [6,7], and here we only briefly outline the method. The reduced Hamiltonian is written as

$$
\mathcal{H}\left(K_{0}, K_{1}, K_{2}, \ldots ; S\right)=-\sum_{\alpha=0}^{\infty} K_{\alpha} S_{\alpha},
$$

where $S$ is a spin configuration, the $K_{\alpha}$ are couplings, and the $S_{\alpha}$ are the conjugate lattice sums over spin products, e.g., $K_{1}$ is the magnetic field and $S_{1}=\sum_{i} s_{i}$ the sum over all spins; $K_{2}$ is the nearest-neighbor coupling and $S_{2}=\sum_{\langle\mathrm{nn}\rangle} s_{i} s_{j}$ the sum over all nearest-neighbor pairs $\left(s_{i}, s_{j}\right)$. A special "coupling" is the background energy density $K_{0} ; S_{0}$ is the number of spins. Application of a block-spin transformation to Monte Carlo generated configurations $S$ leads to configurations $S^{\prime}$ described by a Hamiltonian $\mathcal{H}^{\prime}=\mathcal{H}\left(K_{0}^{\prime}, K_{1}^{\prime}, K_{2}^{\prime}, \ldots ; S^{\prime}\right)$. The renormalized couplings $K_{\alpha}^{\prime}$ are assumed to be analytic functions of the original ones, even at the infinite system critical point. However, this property remains unproven in general, even for $K_{0}^{\prime}$, or the so-called "analytic part" of the transformation.

It is straightforward to calculate, using the Monte Carlo method,

$$
B_{\alpha \beta}^{(i)}=\left\langle\left\langle S_{\alpha}^{\prime} S_{\beta}^{\prime}\right\rangle\right\rangle \equiv\left\langle\left(S_{\alpha}^{\prime}-\left\langle S_{\alpha}^{\prime}\right\rangle\right)\left(S_{\beta}^{\prime}-\left\langle S_{\beta}^{\prime}\right\rangle\right)\right\rangle
$$

and

$$
C_{\alpha \beta}^{(i)}=\left\langle\left\langle S_{\alpha}^{\prime} S_{\beta}\right\rangle\right\rangle \equiv\left\langle\left(S_{\alpha}^{\prime}-\left\langle S_{\alpha}^{\prime}\right\rangle\right)\left(S_{\beta}-\left\langle S_{\beta}\right\rangle\right)\right\rangle .
$$

These lattice sum correlations are related [8] to the linearized transformation

$$
T_{\alpha \beta}=\partial K_{\alpha}^{\prime} / \partial K_{\beta}
$$

via

$$
B_{\alpha \gamma} T_{\gamma \beta}=C_{\alpha \beta} .
$$

The dummy index summation rule applies to Greek indices. The matrix $\mathbf{T}$ is approximated by solving Eq. (5) after truncation to a finite number of couplings. Under iteration of the block-spin transformation, the $K_{\alpha}(\alpha>0)$ are assumed to approach a critical fixed point, where the eigenvalues of $\mathbf{T}$ determine the critical exponents.

Thus, the MCRG method relies on assumptions of (1) analyticity of the transformation, (2) convergence with the dimensionality of the coupling subspace, and (3) convergence to a critical fixed point.

Concerning the third assumption, numerical work involving several subsequent transformations [2-4] suggests that convergence to a fixed point does occur, and is described by an irrelevant exponent $y_{i}$ in the range -0.8 to -1.0 .

In order to investigate the second assumption, the number of couplings $n_{c}$ used in the analyses has increased considerably over the years; from 7 in Ref. [1] to 99 in Ref. [4]. A criterion to distinguish "important" and "less important" couplings was introduced in Ref. [3]. The "importance index" of an $n$-spin coupling is given by $\left(2^{n / 2} \bar{r}\right)^{-1}$, where $\bar{r}$ is the average distance between the spins. This formula accounts for the facts that couplings tend to become less important when more spins are involved and when $\bar{r}$ increases [9]. An ordering according to this index leads to fast apparent convergence [3] with increasing $n_{c}$. On this basis we have restricted the present calculations to 20 even and 15 odd couplings, and indeed we observe good convergence with $n_{c}$ for all results presented here. 
Next, we search for nonanalyticities in the "analytic" part of the transformation when applied to an infinite system. First we investigate if the analytic parts of the susceptibility and the specific heat of the nearest-neighbor model, which are proportional to $T_{011}$ and $T_{022}$, respectively, are bounded at criticality. We express these quantities in derivatives of $\ln Z$ and apply the chain rule:

$$
\begin{aligned}
\frac{\partial^{2} \ln Z}{\partial K_{\alpha} \partial K_{\beta}} & =\frac{\partial}{\partial K_{\alpha}} T_{\gamma \beta} \frac{\partial \ln Z}{\partial K_{\gamma}^{\prime}} \\
& =T_{\gamma \alpha \beta} \frac{\partial \ln Z}{\partial K_{\gamma}^{\prime}}+T_{\gamma \beta} \frac{\partial^{2} \ln Z}{\partial K_{\alpha} \partial K_{\gamma}^{\prime}},
\end{aligned}
$$

where $T_{\gamma \alpha \beta}=\partial T_{\gamma \beta} / \partial K_{\alpha}$. The derivatives of $\ln Z$ can trivially be expressed in connected lattice sum correlations:

$$
\left\langle\left\langle S_{\alpha} S_{\beta}\right\rangle\right\rangle=T_{\gamma \alpha \beta}\left\langle S_{\gamma}^{\prime}\right\rangle+T_{\gamma \beta}\left\langle\left\langle S_{\alpha} S_{\gamma}^{\prime}\right\rangle\right\rangle .
$$

The $T_{\gamma \alpha \beta}$ are the only unknowns in Eq. (7); the correlations follow from the simulation, and the $T_{\gamma \beta}$ from the standard MCRG analysis. There are not enough equations to solve for $T_{0 m m}$, but we can calculate the quantity $A_{m m}=L^{-3} T_{\gamma m m}\left\langle S_{\gamma}^{\prime}\right\rangle$, in which the effect of a possible divergence of $T_{0 \mathrm{~mm}}$ vanishes only in the case of unlikely cancellations. The factor $L^{-3}$ normalizes $S_{\gamma}^{\prime}$ with respect to the system size $L$.

We have done such calculations using the DISP [10,11], a special-purpose computer for Metropolis simulations of Ising models. The transformation is defined by the probability $P\left(s^{\prime}\right)$ of a block spin $s^{\prime}: P\left(s^{\prime}\right)=\exp \left(\omega s^{\prime} s_{b}\right) /$ $2 \cosh \left(\omega s_{b}\right)$, where $s_{b}$ is the sum of the spins in a $2^{3}$ block. It approaches the majority rule for large $\omega$. In the limit of small $\omega$, the block spins become independent and the critical singularity moves to the analytic part [12]. Numerical results for $A_{11}$ and $A_{22}$ did not suggest divergences in the analytic parts of the susceptibility, except where expected: for small $\omega$. Figure 1 shows the numerical results for $A_{11}$ as a function of $\omega$ for $L=8$, 16 , and 32 .

A stricter test uses an explicit calculation of $T_{0 \mathrm{~mm}}$. We apply the chain rule to the second differentiation in $\partial^{3} \ln Z / \partial K_{\alpha} \partial K_{\beta} \partial K_{\gamma}^{\prime}$ and express the derivatives of $\ln Z$ again in connected lattice sum correlations:

$$
\left\langle\left\langle S_{\alpha} S_{\beta} S_{\gamma}^{\prime}\right\rangle\right\rangle=T_{\delta \alpha \beta}\left\langle\left\langle S_{\gamma}^{\prime} S_{\delta}^{\prime}\right\rangle\right\rangle+T_{\delta \beta}\left\langle\left\langle S_{\alpha} S_{\gamma}^{\prime} S_{\delta}^{\prime}\right\rangle\right\rangle .
$$

Choosing $\alpha=\beta=m$ one can solve the unknowns $T_{\delta m m}$ $(\delta>0)$ from the numerical data, and thus isolate the term with $T_{0 m m}$ in Eq. (7). No signs of divergences are seen in the analytic part of the specific heat, except for small $\omega$, as illustrated in Fig. 2.

These results are gratifying, but the transformation may still be weakly singular at the infinite system critical point, or even ill defined [13]. Such problems may be expected if the block-spin transformation fails to lead to a fixed point where the usual corrections to scaling vanish. They should vanish at the fixed point of a well-behaved transformation

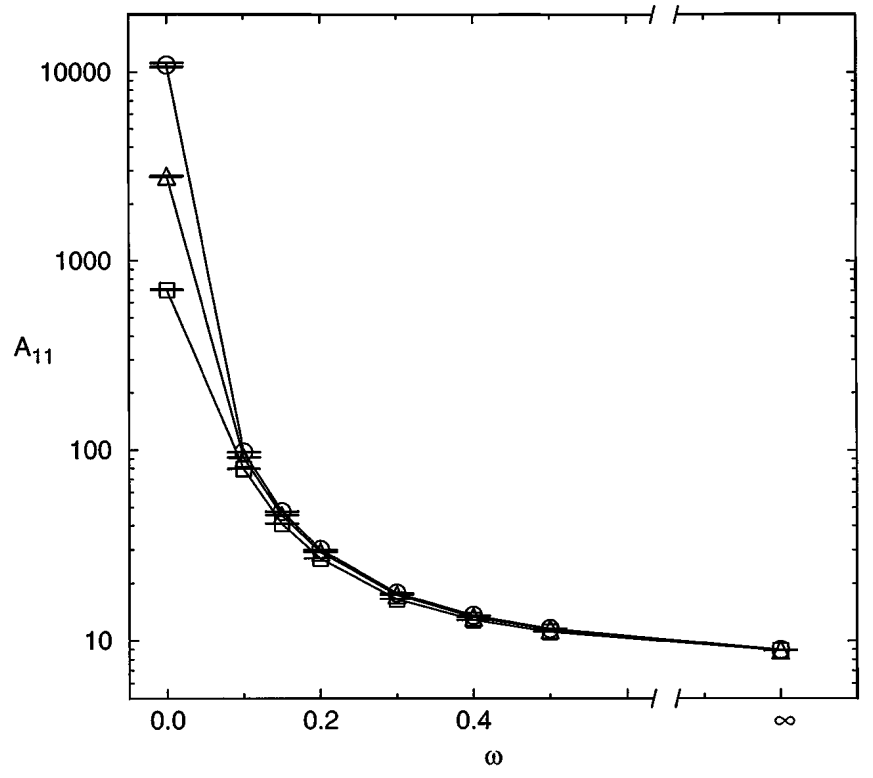

FIG. 1. The quantity $A_{11}$ defined in the text vs the blockspin parameter $\omega$ for finite sizes $L=8(\square), L=16(\triangle)$, and $L=32(\bigcirc)$. The lines are guides to the eye. Signs of a divergence with $L$ appear only for small $\omega$.

[5]. But do they really vanish? This seems doubtful, in particular, when the transformation contains free parameters which move the fixed point over the critical surface. Since the irrelevant fields are absent at a fixed point, any corrections should be due to some other mechanism. Weak singularities associated with corrections to scaling could enter into the renormalized Hamiltonian via a weak nonanalyticity of the transformation.

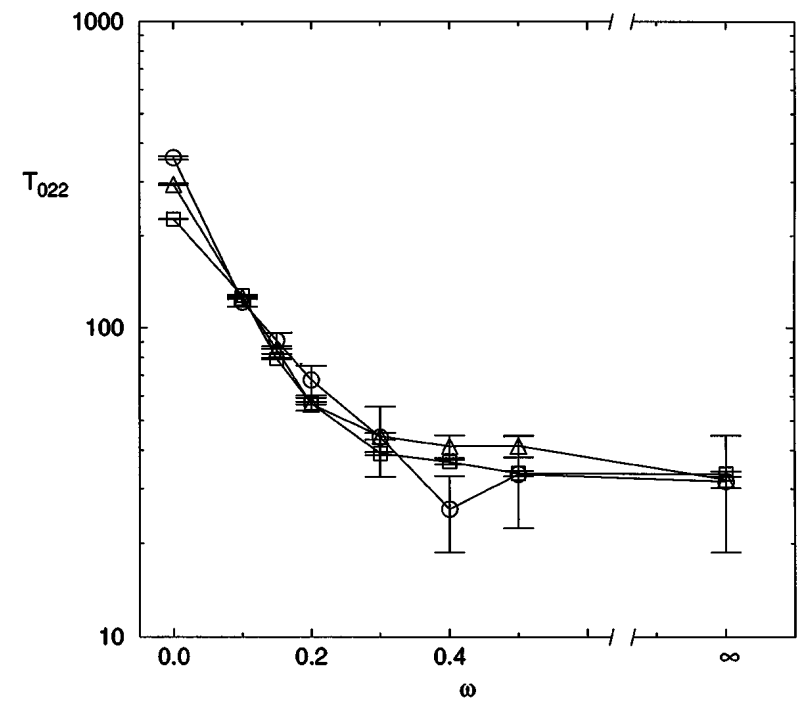

FIG. 2. The quantity $T_{022}$, which is proportional to the analytic part of the specific heat, vs the block-spin parameter $\omega$ for finite sizes $L=8(\square), L=16(\triangle)$, and $L=32(\bigcirc)$. The lines are guides to the eye. Signs of a divergence with $L$ appear only for small $\omega$. 
In order to suppress this problem in MCRG, we propose the following strategy, which is applicable in a more general context than the Ising model: (1) The Hamiltonian used to generate the Monte Carlo configurations is chosen such that the corrections to scaling are small. (2) The transformation is chosen such that the fixed point is close to the Hamiltonian mentioned. To this purpose we included, in addition to nearest-neighbor couplings $K_{2}=K_{\mathrm{nn}}$, second and third neighbor couplings $K_{2 n}$ and $K_{3 n}$ in the Monte Carlo simulation [14], and optimized the ratio between the couplings, and the block-spin parameter $\omega$.

First we used a Monte Carlo Hamiltonian with $K_{2 n}=0$ and $K_{3 n} / K_{\mathrm{nn}}=0.4$, for which the corrections to scaling are small $[15,16]$. Then the convergence to the fixed point, as apparent from the MCRG results for the eigenvalues of $T_{\alpha \beta}$, becomes optimal for $\omega=0.4$. This is close to a variational value found by Kadanoff, Houghton, and Yalabik [17]. The difference between the Monte Carlo and the fixed-point Hamiltonian follows, in a linear approximation [2,6], from the difference between $\left\langle S_{\alpha}\right\rangle$ and $\left\langle S_{\alpha}^{\prime}\right\rangle$ as determined from separate simulations of systems with compatible sizes. This calculation was done in the coupling subspace $\left(K_{\mathrm{nn}}, K_{2 n}, K_{3 n}\right)$.

A second approximation of the $\omega=0.4$ fixed point was found by using a Monte Carlo Hamiltonian close to the first approximation. The fixed point was thus estimated $\left(K_{\mathrm{nn}}, K_{2 n}, K_{3 n}\right)=(0.1109,0.03308,0.01402)$. A finite-size scaling analysis of Monte Carlo results [15] was used to determine the critical point more accurately: $\left(K_{\mathrm{nn}}, K_{2 n}, K_{3 n}\right)=(0.1114448,0.0332520,0.0140925)$, with a relative accuracy of $2 \times 10^{-5}$. This analysis showed that the corrections to scaling in the Binder cumulant [18] are about 6 times smaller than for the nearest-neighbor Hamiltonian.

The bulk of the MCRG calculations took place at the estimated critical point, using system sizes $L=32,16$, and 8 and lengths of $10^{8}, 2 \times 10^{7}$, and $10^{7}$ sweeps, respectively. The sensitivity to a variation in $K_{\mathrm{nn}}$ was estimated from additional runs at $K_{\mathrm{nn}}=0.1114336$ and 0.1114560 .

Further details including the ordering of the couplings according to the importance index are contained in Ref. [3]. Table I lists the resulting estimates for the exponents $y_{T}$ and $y_{H}$, as determined from the largest

TABLE I. Numerical results for the renormalization exponents $y_{T}$ and $y_{H}$, obtained after $n$ block-spin transformations of a system of size $L$.

\begin{tabular}{ccccc}
\hline \hline Exponent & $n$ & \multicolumn{1}{c}{$L=32$} & \multicolumn{1}{c}{$L=16$} & $L=8$ \\
\hline$y_{T}$ & 1 & $1.5885(3)$ & $1.5885(6)$ & $1.5868(8)$ \\
$y_{T}$ & 2 & $1.5852(5)$ & $1.5829(9)$ & \\
$y_{T}$ & 3 & $1.5829(9)$ & & \\
$y_{H}$ & 1 & $2.48492(4)$ & $2.48500(7)$ & $2.48521(22)$ \\
$y_{H}$ & 2 & $2.48309(11)$ & $2.48327(25)$ & \\
$y_{H}$ & 3 & $2.48219(27)$ & & \\
\hline \hline
\end{tabular}

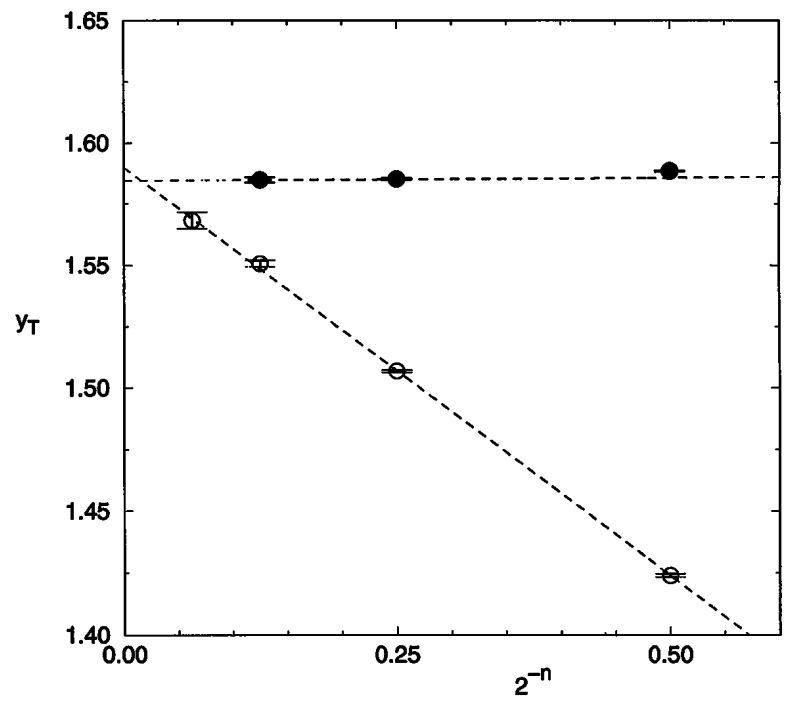

FIG. 3. The convergence of the temperature exponent $y_{T}$ with increasing number of iterations $n$ of the block-spin transformation. Results are shown for the present MCRG calculations (O) and for those reported in Ref. [3] $(\bigcirc)$.

eigenvalues of the $T_{\alpha \beta}$ matrix. Statistical errors were found by dividing the runs in 50 subruns. Finite-size and renormalization (approach of the fixed point) effects were determined with the procedures described, e.g., in Ref. [2]. The convergence of $y_{T}$ and $y_{H}$ vs $2^{-n}$ is, after correction for the finite-size effect, shown in Figs. 3 and 4. For comparison we include results from Ref. [3], which used the standard nearest-neighbor Hamiltonian and the majority rule. Extrapolation of the data for $L=$ 32 yields our final estimates for the critical exponents: $y_{H}=2.481(1)$ and $y_{T}=1.585(3)$. These results provide

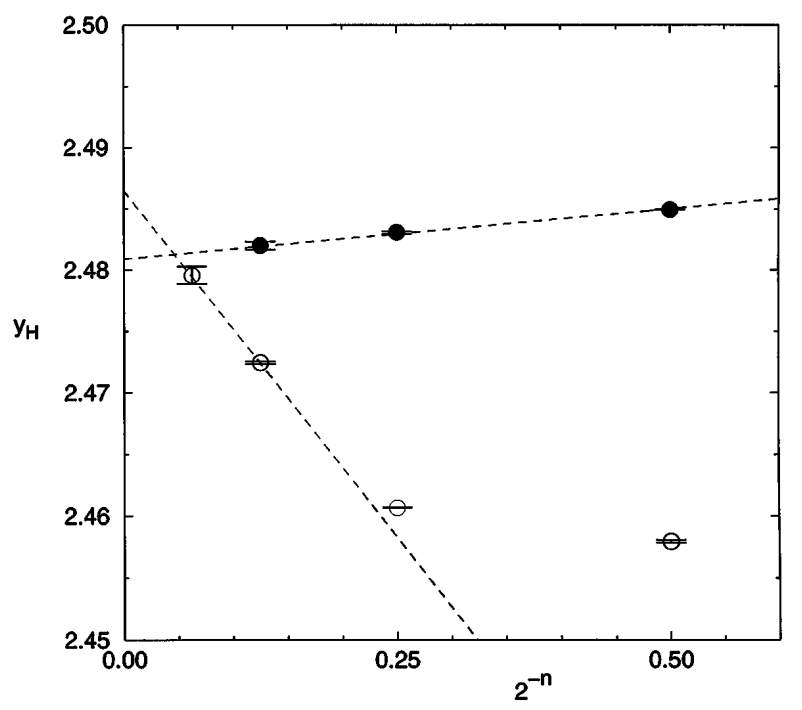

FIG. 4. The convergence of the magnetic exponent $y_{H}$ with increasing number of iterations $n$ of the block-spin transformation. Results are shown for the present MCRG calculations and for those reported in Ref. [3] (O). 
a significant improvement over previous MCRG analyses, not only concerning the statistical errors, but also the consistency with other results for the Ising universality class which are summarized, e.g., in Ref. [16]. The precise agreement of the renormalization results with those obtained by finite-size scaling confirms the validity of hyperscaling [19].

Earlier attempts to accelerate the convergence used optimized transformations [20-25]. The present work uses this idea combined with a Hamiltonian with suppressed corrections to scaling. This restores the consistency with the usual renormalization assumptions, and leads to a much improved apparent convergence to the fixed point. This rapid convergence eliminates the necessity of timeconsuming simulations of large system sizes. The error due to the uncertainty margin of the exponent describing the renormalization effect practically vanishes. Further improvements of the MCRG method may be possible by the introduction of more adjustable parameters in the block-spin transformation, so that its fixed point can be moved to a point with even smaller corrections to scaling.

It is a pleasure to thank A. C. D. van Enter and B. Nienhuis for valuable discussions. This work is part of the research program of the "Stichting voor Fundamenteel Onderzoek der Materie (FOM)," which is financially supported by the "Nederlandse Organisatie voor Wetenschappelijk Onderzoek (NWO)."

[1] H. W. J. Blöte and R. H. Swendsen, Phys. Rev. B 20, 2077 (1979).

[2] G.S. Pawley, R.H. Swendsen, D. J. Wallace, and K. G. Wilson, Phys. Rev. B 29, 4030 (1984).

[3] H. W. J. Blöte, A. Compagner, J. H. Croockewit, Y. T. J. C. Fonk, J. R. Heringa, A. Hoogland, T.S. Smit, and A. L. van Willigen, Physica (Amsterdam) 161A, 1 (1989).

[4] C.F. Baillie, R. Gupta, K. A. Hawick, and G. S. Pawley, Phys. Rev. B 45, 10438 (1992).
[5] M. E. Fisher and M. Randeria, Phys. Rev. Lett. 56, 2332 (1986).

[6] R. H. Swendsen, in Real Space Renormalization, edited by T.W. Burkhardt and J. M. J. van Leeuwen (Springer, Berlin, 1982).

[7] R. Gupta, J. Appl. Phys. 61, 3605 (1987).

[8] R. H. Swendsen, Phys. Rev. Lett. 42, 859 (1979).

[9] M. R. Dudek, J. M. J. van Leeuwen, and H. W. J. Blöte, Physica (Amsterdam) 147A, 344 (1987), and references therein.

[10] A. Hoogland, J. Spaa, B. Selman, and A. Compagner, J. Comp. Phys. 51, 250 (1983).

[11] A. Hoogland, A. Compagner, and H.W.J. Blöte, in Special Purpose Computers (Computational Techniques), edited by B.J. Alder (Academic, New York, 1988), Vol. 5.

[12] Th. Niemeijer and J. M. J. van Leeuwen, in Phase Transitions and Critical Phenomena, edited by C. Domb and M. S. Green (Academic, New York, 1971), Vol. 6.

[13] A. C. D. van Enter, R. Fernández, and A. D. Sokal, J. Stat. Phys. 72, 879 (1993).

[14] S. H. Shenker and J. Tobochnik, Phys. Rev. B 22, 4462 (1980).

[15] H. W. J. Blöte, J. R. Heringa, A. Hoogland, E. W. Meyer, and A. L. van Willigen (to be published).

[16] H. W. J. Blöte, E. Luijten, and J. R. Heringa, J. Phys. A 28, 6289 (1995).

[17] L. P. Kadanoff, A. Houghton, and M. C. Yalabik, J. Stat. Phys. 14, 171 (1976).

[18] K. Binder, Z. Phys. B 43, 119 (1981).

[19] G. A. Baker and N. Kawashima, Phys. Rev. Lett. 75, 994 (1995).

[20] R. H. Swendsen, Phys. Rev. Lett. 52, 2321 (1984).

[21] R. Gupta and R. Cordery, Phys. Lett. 105A, 415 (1984).

[22] A. Hasenfratz, P. Hasenfratz, U. Heller, and F. Karsch, Phys. Lett. 140B, 76 (1984).

[23] H. Gausterer and C. B. Lang, Phys. Lett. B 186, 103 (1987).

[24] A. Bennett, J. Phys. A 20, 4891 (1987).

[25] T. S. Smit, J. R. Heringa, H. W. J. Blöte, A. Compagner, Y. T. J. C. Fonk, and A. Hoogland, Int. J. Mod. Phys. C 2, 487 (1991). 